\title{
ON AN IMPLICIT HIERARCHICAL FIXED POINT APPROACH TO VARIATIONAL INEQUALITIES
}

\author{
FILOMENA CIANCIARUSO, VITTORIO COLAO, LUIGI MUGLIA and \\ HONG-KUN XU ${ }^{凶}$
}

(Received 4 September 2008)

\begin{abstract}
Moudafi and Maingé [Towards viscosity approximations of hierarchical fixed-point problems, Fixed Point Theory Appl. (2006), Art. ID 95453, 10pp] and Xu [Viscosity method for hierarchical fixed point approach to variational inequalities, Taiwanese J. Math. 13(6) (2009)] studied an implicit viscosity method for approximating solutions of variational inequalities by solving hierarchical fixed point problems. The approximate solutions are a net $\left(x_{s, t}\right)$ of two parameters $s, t \in(0,1)$, and under certain conditions, the iterated $\lim _{t \rightarrow 0} \lim _{s \rightarrow 0} x_{s, t}$ exists in the norm topology. Moudafi, Maingé and Xu stated the problem of convergence of $\left(x_{s, t}\right)$ as $(s, t) \rightarrow(0,0)$ jointly in the norm topology. In this paper we further study the behaviour of the net $\left(x_{s, t}\right)$; in particular, we give a negative answer to this problem.
\end{abstract}

2000 Mathematics subject classification: primary 49J40; secondary 47J20, 47H09.

Keywords and phrases: implicit method, variational inequality, hierarchical fixed point, nonexpansive mapping, projection.

\section{Introduction and preliminaries}

A useful method for solving ill-posed nonlinear problems is to substitute the originally ill-posed problem by a family of regularized (well-posed) problems. A particular (viscosity) solution of the original problem is then obtained as limit of the solutions of the regularized problems. In $[4,7,10]$ the authors used this idea to provide a viscosity method for solving variational inequality problems via a hierarchical fixed point approach.

Let $T, V$ be two nonexpansive mappings from $C$ to $C$, where $C$ is a closed convex subset of a Hilbert space $H$. Consider the variational inequality (VI) of finding hierarchically a fixed point of $T$ with respect to $V$, that is,

$$
\text { Find } x^{*} \in \operatorname{Fix}(T) \quad \text { such that }\left\langle x^{*}-V x^{*}, y-x^{*}\right\rangle \geq 0, y \in \operatorname{Fix}(T) \text {. }
$$

The first three authors were supported in part by Ministero dell'Universitá e della Ricerca of Italy. The fourth author was supported in part by NSC 97-2628-M-110-003-MY3 (Taiwan).

(c) 2009 Australian Mathematical Society 0004-9727/2009 \$16.00 
Equivalently, $x^{*}=P_{\mathrm{Fix}(T)} V x^{*}$; that is, $x^{*}$ is a fixed point of the nonexpansive mapping $P_{\mathrm{Fix}(T)} V$, where $P_{K}$ denotes the metric projection from $H$ on a closed convex subset $K$ of $H$. The VI (1.1) covers several topics investigated in the literature (see $[1,3,5,6,8,11,12]$ and the references cited therein).

Let $S$ denote the solution set of (1.1) and assume throughout the rest of this paper that $S \neq \emptyset$. Note that $S=\operatorname{Fix}\left(P_{\operatorname{Fix}(T)} V\right)$. We also adopt the following notation: $x_{n} \rightarrow x$ means that $\left(x_{n}\right)$ converges to $x$ in the norm topology; $x_{n} \rightarrow x$ means that $\left(x_{n}\right)$ converges to $x$ in the weak topology.

Let $f: C \rightarrow C$ be a $\rho$-contraction and define, for $s, t \in(0,1)$, two mappings $W_{t}$ and $f_{s, t}$ by

$$
W_{t}=t V+(1-t) T, \quad f_{s, t}=s f+(1-s) W_{t} .
$$

It is easy to verify that $W_{t}$ is nonexpansive and $f_{s, t}$ is a $[1-(1-\rho) s]$-contraction.

Let $x_{s, t}$ be the unique fixed point of $f_{s, t}$, that is, the unique solution of the fixed point equation

$$
x_{s, t}=s f\left(x_{s, t}\right)+(1-s) W_{t} x_{s, t} .
$$

Moudafi and Maingé [7] initiated the investigation of the iterated behaviour of the net $\left(x_{s, t}\right)$ as $s \rightarrow 0$ firstly and $t \rightarrow 0$ secondly. They make the following assumptions:

(A1) for each $t \in(0,1)$, the fixed point set $\operatorname{Fix}\left(W_{t}\right)$ of $W_{t}$ is nonempty and the set $\left\{\operatorname{Fix}\left(W_{t}\right): 0<t<1\right\}=\bigcup_{t \in(0,1)} \operatorname{Fix}\left(W_{t}\right)$ is bounded; and (A2) $\emptyset \neq S \subset\|\cdot\|-\lim _{\inf _{t \rightarrow 0}} \operatorname{Fix}\left(W_{t}\right):=\left\{z: \exists z_{t} \in \operatorname{Fix}\left(W_{t}\right)\right.$ such that $\left.z_{t} \rightarrow z\right\}$.

Moudafi and Maingé [7] (see also [9]) proved that, for each fixed $t \in(0,1)$, as $s \rightarrow 0$, $x_{s, t} \rightarrow x_{t}$; moreover, as $t \rightarrow 0, x_{t} \rightarrow x_{\infty}$ which is the unique solution to the VI

$$
x_{\infty} \in S, \quad\left\langle x_{\infty}-f\left(x_{\infty}\right), x-x_{\infty}\right\rangle \geq 0, x \in S .
$$

The following theorem, due to $\mathrm{Xu}$ [10], improves the Moudafi-Maingé result since he proves that $\left(x_{t}\right)$ actually strongly converges to $x_{\infty}$. Moreover, $\mathrm{Xu}$ does not need the boundedness assumption of the set $\bigcup_{t \in(0,1)} \operatorname{Fix}\left(W_{t}\right)$.

THEOREM 1.1. [10] Let the above assumption (A2) hold. Assume also that, for each $t \in(0,1), \operatorname{Fix}\left(W_{t}\right)$ is nonempty (but not necessarily bounded). Then the strong $\lim _{s \rightarrow 0} x_{s, t}=: x_{t}$ exists for each $t \in(0,1)$. Moreover, the strong $\lim _{t \rightarrow 0} x_{t}=: x_{\infty}$ exists and solves the VI (1.3). Hence, for each null sequence $\left(s_{n}\right)$ in $(0,1)$, there is another null sequence $\left(t_{n}\right)$ in $(0,1)$ such that $x_{s_{n}, t_{n}} \rightarrow x_{\infty}$, as $n \rightarrow \infty$.

In $[7,10]$, the authors stated the problem of the convergence of $\left(x_{s, t}\right)$ when $(s, t) \rightarrow(0,0)$ jointly. In this paper, we further investigate the behaviour of the net $\left(x_{s, t}\right)$ along the curve $t=t(s)$ and our results point to a negative answer to this problem. Specifically, we prove that:

(i) $\quad$ if $t(s)=O(s)$, as $s \rightarrow 0$, then $x_{s, t(s)} \rightarrow z_{\infty} \in \operatorname{Fix}(T)$; and

(ii) if $t(s) / s \rightarrow \infty$, as $s \rightarrow 0$, then $x_{s, t(s)} \rightarrow x_{\infty} \in S$. 
We next include two lemmas which are pertinent to the proof of many convergence results of iterative methods. Let $H$ be a real Hilbert space and $C$ be a nonempty closed convex of $H$. Recall that the metric projection, $P_{C}$, from $H$ onto $C$, assigns to each $x \in H$ a unique point $P_{C} x$ in $C$ with the property

$$
\left\|x-P_{C} x\right\|=\inf _{y \in C}\|x-y\| .
$$

LeMma 1.2. Given $x \in H$ and $z \in C$, then $z=P_{C} x$ if and only if

$$
\langle x-z, y-z\rangle \leq 0 \quad \forall y \in C .
$$

LEMMA 1.3 ([2] Demiclosedness principle). If $T: C \rightarrow C$ is a nonexpansive mapping with $\operatorname{Fix}(T) \neq \emptyset$, then the mapping $(I-T)$ is demiclosed; that is, if a sequence $\left(x_{n}\right)$ in $C$ is weakly convergent to $x$ and if the sequence $\left((I-T) x_{n}\right)$ is strongly convergent to $y$, then $(I-T) x=y$.

\section{On convergence of $\left(x_{s, t}\right)_{s, t \in(0,1)}$}

In this section we study the convergence of the net $\left(x_{s, t}\right)$ along the curve $t=t(s)=$ : $t_{s}$, where $t_{s}=O(s)$, as $s \rightarrow 0$.

THEOREM 2.1. Let $H$ be a real Hilbert space and let $C$ be a closed convex subset of $H$. Let $V, T: C \rightarrow C$ be nonexpansive mappings with $\operatorname{Fix}(T) \neq \emptyset$. Let $f: C \rightarrow C$ be a $\rho$-contraction with $\rho \in[0,1)$. Assume that $t_{s}=O(s)$, as $s \rightarrow 0$, and let $l=$ $\lim _{\sup _{s \rightarrow 0}}\left(t_{s} / s\right)$. Then the net $\left(x_{s, t_{s}}\right)_{s \in(0,1)}$ defined by

$$
x_{s, t_{s}}=s f\left(x_{s, t_{s}}\right)+(1-s) W_{t_{s}} x_{s, t_{s}}
$$

strongly converges to $z_{\infty} \in \operatorname{Fix}(T)$ which is the unique solution of the VI

$$
z_{\infty} \in \operatorname{Fix}(T), \quad\left\langle[(I-f)+l(I-V)] z_{\infty}, x-z_{\infty}\right\rangle \geq 0, x \in \operatorname{Fix}(T) .
$$

PROOF. We first note that the VI (2.2) has a unique solution, due to the fact that the operator $(I-f)+l(I-V)$ is strongly monotone. The proof is divided into two steps.

The first step is to prove that the net $\left(x_{s, t_{s}}\right)_{s \in(0,1)}$ is bounded. Let $z \in \operatorname{Fix}(T)$; then, from (2.1),

$$
\begin{aligned}
\left\|x_{s, t_{s}}-z\right\|^{2}= & \left\langle x_{s, t_{s}}-z, x_{s, t_{s}}-z\right\rangle \\
= & s\left\langle f\left(x_{s, t_{s}}\right)-z, x_{s, t_{s}}-z\right\rangle+(1-s)\left\langle W_{t_{s}} x_{s, t_{s}}-z, x_{s, t_{s}}-z\right\rangle \\
= & s\left[\left\langle f\left(x_{s, t_{s}}\right)-f(z), x_{s, t_{s}}-z\right\rangle+\left\langle f(z)-z, x_{s, t_{s}}-z\right\rangle\right] \\
& \quad+(1-s)\left[\left\langle W_{t_{s}} x_{s, t_{s}}-W_{t_{s}} z, x_{s, t_{s}}-z\right\rangle+\left\langle W_{t_{s}} z-z, x_{s, t_{s}}-z\right\rangle\right] \\
& \quad s \rho\left\|x_{s, t_{s}}-z\right\|^{2}+s\left\langle f(z)-z, x_{s, t_{s}}-z\right\rangle \\
& \quad+(1-s)\left\|x_{s, t_{s}}-z\right\|^{2}+t_{s}(1-s)\left\langle V z-z, x_{s, t_{s}}-z\right\rangle .
\end{aligned}
$$


Simplifying, we obtain

$$
\left\|x_{s, t_{s}}-z\right\|^{2} \leq \frac{1}{1-\rho}\left[\left\langle f(z)-z, x_{s, t_{s}}-z\right\rangle+\frac{t_{s}(1-s)}{s}\left\langle V z-z, x_{s, t_{s}}-z\right\rangle\right] .
$$

In particular,

$$
\left\|x_{s, t_{s}}-z\right\| \leq \frac{1}{1-\rho}\left[\|f(z)-z\|+\frac{t_{s}}{s}\|V z-z\|\right] .
$$

Since $t_{s}=O(s)$, as $s \rightarrow 0,(2.4)$ implies the boundedness of $\left(x_{s, t_{s}}\right)$ and the first step is proved.

The second step is to prove that the net $x_{s, t_{s}} \rightarrow z_{\infty} \in \operatorname{Fix}(T)$, as $s \rightarrow 0$, where $z_{\infty}$ is the unique solution of the VI (2.2). We observe that

$$
\left\|x_{s, t_{s}}-T x_{s, t_{s}}\right\| \leq s\left\|f\left(x_{s, t_{s}}\right)\right\|+(1-s) t_{s}\left\|V x_{s, t_{s}}\right\|+\left(s+t_{s}-s t_{s}\right) \mid\left\|T x_{s, t_{s}}\right\| .
$$

Since $\left(x_{s, t_{s}}\right)$ is bounded when $s \rightarrow 0$ (hence $t_{s} \rightarrow 0$ ), we find that

$$
\left\|x_{s, t_{s}}-T x_{s, t_{s}}\right\| \rightarrow 0
$$

We now claim that $\left(x_{s, t_{s}}\right)_{s \in(0,1)}$ is relatively compact as $s \rightarrow 0$ in the norm topology. To see this, assume $\left(s_{n}\right)$ is null sequence in $(0,1)$. Without loss of generality, we may assume that $x_{s_{n}, t_{s_{n}}} \rightarrow \widehat{x}$ which implies from (2.5) and Lemma 1.3 that $\widehat{x} \in \operatorname{Fix}(T)$. We thus immediately get from (2.3) that $x_{s_{n}, t_{s_{n}}} \rightarrow \widehat{x}$.

We next further claim that $\widehat{x}=z_{\infty}$, the unique solution to the VI (2.2), which then completes the proof. Indeed, observing

$$
(I-f) x_{s, t}=-\frac{1-s}{s}\left(x_{s, t}-W_{t} x_{s, t}\right)=-\frac{1-s}{s}\left[t(I-V) x_{s, t}+(1-t) T x_{s, t}\right],
$$

we deduce that, for $z \in \operatorname{Fix}(T)$,

$$
\begin{aligned}
\left\langle(I-f) x_{s, t}, x_{s, t}-z\right\rangle=-\frac{1-s}{s}\left[t\left\langle(I-V) x_{s, t}, x_{s, t}-z\right\rangle\right. & \\
& \left.+(1-t)\left\langle(I-T) x_{s, t}, x_{s, t}-z\right\rangle\right] .
\end{aligned}
$$

However, since

$$
\left\langle(I-T) x_{s, t}, x_{s, t}-z\right\rangle=\left\langle(I-T) x_{s, t}-(I-T) z, x_{s, t}-z\right\rangle \geq 0,
$$

we obtain

$$
\left\langle(I-f) x_{s, t}, x_{s, t}-z\right\rangle \leq-\frac{t(1-s)}{s}\left\langle(I-V) x_{s, t}, x_{s, t}-z\right\rangle .
$$

Now since $x_{s_{n}, t_{s_{n}}} \rightarrow \widehat{x}$, setting $s=s_{n}$ and $t=t_{s_{n}}$ in (2.6) and letting $n \rightarrow \infty$, we immediately see that $\widehat{x}$ satisfies the VI (2.2) and therefore we must have $\widehat{x}=z_{\infty}$ since $z_{\infty}$ is the unique solution of (2.2). 
REMARK 2.2. (i) If $t_{s}=o(s)$ (that is, $l=0$ ), then the above argument shows that the net $\left(x_{s, t_{s}}\right)$ actually converges in norm to the unique solution of the VI

$$
x_{\infty} \in \operatorname{Fix}(T), \quad\left\langle x_{\infty}-f\left(x_{\infty}\right), p-x_{\infty}\right\rangle \geq 0, p \in \operatorname{Fix}(T),
$$

which is also the unique fixed point of the contraction $P_{\operatorname{Fix}(T)} f, x_{\infty}=\left(P_{\operatorname{Fix}(T)} f\right) x_{\infty}$. This is Theorem 3.3 in $\mathrm{Xu}[10]$.

(ii) The net $\left(x_{s, t}\right)_{s, t \in(0,1)}$ does not converge, in general, as $(s, t) \rightarrow(0,0)$ jointly, to the unique solution $x_{\infty} \in S$ of the VI (1.3). As a matter of fact, if $\left(x_{s, t}\right)_{s, t \in(0,1)}$ converged to $x_{\infty}$ jointly as $(s, t) \rightarrow(0,0)$, then (by (2.7) we would have the relation and (1.3))

$$
x_{\infty}=P_{S} f\left(x_{\infty}\right)=P_{\operatorname{Fix}(T)} f\left(x_{\infty}\right)
$$

for all $\rho$-contractions $f$. This implies that $S=\operatorname{Fix}(T)$ which is not true, in general.

(iii) Consider the case of $l>0$. If $x_{\infty}$, the unique solution of (2.7), belongs to $S$, then, clearly, $x_{\infty}=z_{\infty}$. If $x_{\infty} \notin S$, the following example shows that there are, in general, no links among $z_{\infty}, S$ and $x_{\infty}$. Take

$$
C=[0,1], \quad T=I, \quad f(x)=\frac{x}{2}, \quad V(x)=1-x, \quad l=1 .
$$

The unique solution $x_{\infty}$ of the VI

$$
x_{\infty} \in[0,1], \quad\left\langle x_{\infty}-f\left(x_{\infty}\right), z-x_{\infty}\right\rangle \geq 0, z \in[0,1],
$$

is $x_{\infty}=0$; the unique solution $z_{\infty}$ of the VI

$$
x_{\infty} \in[0,1], \quad\left\langle\left(z_{\infty}-f\left(z_{\infty}\right)\right)+\left(z_{\infty}-V z_{\infty}\right), z-z_{\infty}\right\rangle \geq 0, z \in[0,1],
$$

is $z_{\infty}=\frac{2}{5}$, and the set $S$ of the solutions of the VI

$$
x \in[0,1], \quad\langle x-V x, z-x\rangle \geq 0, z \in[0,1],
$$

is the singleton $\{1 / 2\}$.

\section{The case $l=\infty$}

In this section we examine the convergence of the net $\left(x_{s, t_{s}}\right)_{s \in(0,1)}$ along the curve where $t_{s} / s \rightarrow \infty$, as $s \rightarrow 0$. We shall prove that the net converges strongly to a point $x_{\infty} \in S$ which is the unique solution of the VI (1.3).

THEOREM 3.1. Let $H$ be a real Hilbert space and let $C$ be a closed convex subset of $H$. Assume that $V, T: C \rightarrow C$ are nonexpansive mappings with $\operatorname{Fix}(T) \neq \emptyset$ and $f: C \rightarrow C$ is a $\rho$-contraction with $\rho \in[0,1)$. Assume the condition (A2) in Section 1. Let $t_{s}=t(s)$ satisfy $\lim _{s \rightarrow 0} t_{s} / s=\infty$. Then the net $\left(x_{s, t_{s}}\right)_{s \in(0,1)}$ defined by

$$
x_{s, t_{s}}=s f\left(x_{s, t_{s}}\right)+(1-s) W_{t_{s}} x_{s, t_{s}}
$$

strongly converges to $x_{\infty} \in S$ which is the unique solution of the VI (1.3). 
PROOF. The proof is divided into three steps, the first of which is to prove the boundedness of $\left(x_{s, t_{s}}\right)_{s \in(0,1)}$. Let $z \in S$. By condition (A2) there exists $p_{s} \in \operatorname{Fix}\left(W_{s}\right)$ such that $p_{s} \rightarrow z$ as $s \rightarrow 0$. We then derive that

$$
\begin{aligned}
\left\|x_{s, t_{s}}-p_{s}\right\|^{2}= & \left\|s\left(f\left(x_{s, t_{s}}\right)-f\left(p_{s}\right)\right)+s\left(f\left(p_{s}\right)-p_{s}\right)+(1-s)\left(W_{t_{s}} x_{s, t_{s}}-p_{s}\right)\right\|^{2} \\
\leq & \left\|s\left(f\left(x_{s, t_{s}}\right)-f\left(p_{s}\right)\right)+(1-s)\left(W_{t_{s}} x_{s, t_{s}}-p_{s}\right)\right\|^{2} \\
& \quad+2 s\left\langle f\left(p_{s}\right)-p_{s}, x_{s, t_{s}}-p_{s}\right\rangle \\
\leq & s\left\|f\left(x_{s, t_{s}}\right)-f\left(p_{s}\right)\right\|^{2}+(1-s)\left\|W_{t_{s}} x_{s, t_{s}}-p_{s}\right\|^{2} \\
& \quad+2 s\left\langle f\left(p_{s}\right)-p_{s}, x_{s, t_{s}}-p_{s}\right\rangle \\
\leq & \left(1-\left(1-\rho^{2}\right) s\right)\left\|x_{s, t_{s}}-p_{s}\right\|^{2}+2 s\left\langle f\left(p_{s}\right)-p_{s}, x_{s, t_{s}}-p_{s}\right\rangle .
\end{aligned}
$$

It follows that

$$
\left\|x_{s, t_{s}}-p_{s}\right\|^{2} \leq \frac{2}{1-\rho^{2}}\left\langle f\left(p_{s}\right)-p_{s}, x_{s, t_{s}}-p_{s}\right\rangle .
$$

This implies immediately that

$$
\left\|x_{s, t_{s}}-p_{s}\right\| \leq \frac{2}{1-\rho^{2}}\left\|f\left(p_{s}\right)-p_{s}\right\| .
$$

From (3.3) the boundedness of $\left(x_{s, t_{s}}\right)_{s \in(0,1)}$ follows since $\left\{p_{s}\right\}$ is bounded.

The second step is to prove that the set of weak cluster points of $\left(x_{s, t_{s}}\right)_{s \in(0,1)}$, $\omega_{w}\left(x_{s, t_{s}}\right)$, is a subset of $S$; moreover, $\omega_{w}\left(x_{s, t_{s}}\right)=\omega_{s}\left(x_{s, t_{s}}\right)$. First observe that the boundedness of $\left(x_{s, t_{s}}\right),(2.5)$, and Lemma 1.3 imply that $\omega_{w}\left(x_{s, t_{s}}\right) \subset \operatorname{Fix}(T)$.

Now let $w \in \omega_{w}\left(x_{s, t_{s}}\right)$ and assume that $x_{n}:=x_{s_{n}, t_{s_{n}}} \rightarrow w$, where $s_{n} \rightarrow 0$. For convenience, we write $t_{n}=t_{s_{n}}$ for all $n$; thus, $t_{n} / s_{n} \rightarrow \infty$ as $n \rightarrow \infty$. Noticing that

$$
x_{n}=s_{n} f\left(x_{n}\right)+\left(1-s_{n}\right)\left[t_{n} V x_{n}+\left(1-t_{n}\right) T x_{n}\right],
$$

we derive that, for each fixed $\widehat{x} \in \operatorname{Fix}(T)$ and for a constant $M \geq \sup _{n}\left\{\| f\left(x_{n}\right)-\right.$ $\left.\widehat{x}\|\| x_{n}-\widehat{x} \|\right\}$,

$$
\begin{aligned}
&\left\|x_{n}-\widehat{x}\right\|^{2}= s_{n}\left\langle f\left(x_{n}\right)-\widehat{x}, x_{n}-\widehat{x}\right\rangle \\
& \quad+\left(1-s_{n}\right)\left(t_{n}\left\langle V x_{n}-\widehat{x}, x_{n}-\widehat{x}\right\rangle+\left(1-t_{n}\right)\left\langle T x_{n}-\widehat{x}, x_{n}-\widehat{x}\right\rangle\right) \\
&= s_{n}\left\langle f\left(x_{n}\right)-\widehat{x}, x_{n}-\widehat{x}\right\rangle+\left(1-s_{n}\right) t_{n}\left\langle V \widehat{x}-\widehat{x}, x_{n}-\widehat{x}\right\rangle \\
& \quad+\left(1-s_{n}\right)\left[t_{n}\left\langle V x_{n}-V \widehat{x}, x_{n}-\widehat{x}\right\rangle+\left(1-t_{n}\right)\left\langle T x_{n}-T \widehat{x}, x_{n}-\widehat{x}\right\rangle\right] \\
& \leq\left\|x_{n}-\widehat{x}\right\|^{2}+\left(1-s_{n}\right) t_{n}\left\langle V \widehat{x}-\widehat{x}, x_{n}-\widehat{x}\right\rangle+s_{n} M .
\end{aligned}
$$

It follows that

$$
\left\langle(I-V) \widehat{x}, x_{n}-\widehat{x}\right\rangle \leq \frac{s_{n} M}{\left(1-s_{n}\right) t_{n}} \rightarrow 0
$$

as $s_{n} / t_{n} \rightarrow 0$. But $x_{n} \rightarrow w$, and we get

$$
\langle(I-V) \widehat{x}, w-\widehat{x}\rangle \leq 0, \quad \widehat{x} \in \operatorname{Fix}(T) .
$$


Upon replacing the $\hat{x}$ in (3.4) with $w+\gamma(\tilde{x}-w) \in \operatorname{Fix}(T)$, where $\gamma \in(0,1)$ and $\tilde{x} \in \operatorname{Fix}(T)$, we get

$$
\langle(I-V)(w+\gamma(\tilde{x}-w)), w-\tilde{x}\rangle \leq 0 .
$$

Letting $\gamma \rightarrow 0$, we obtain the VI

$$
\langle(I-V) w, w-\tilde{x}\rangle \leq 0, \quad \tilde{x} \in \operatorname{Fix}(T) .
$$

Therefore, $w \in S$.

Next using condition (A2) again, we have a sequence $p_{n} \in \operatorname{Fix}\left(W_{t_{n}}\right)$ such that $p_{n} \rightarrow w$. Then in relation (3.2) we replace $z$ and $p_{s}$ with $w$ and $p_{n}$, respectively, to get

$$
\left\|x_{n}-p_{n}\right\|^{2} \leq \frac{2}{1-\rho^{2}}\left\langle f\left(p_{n}\right)-p_{n}, x_{n}-p_{n}\right\rangle .
$$

Now since $f\left(p_{n}\right)-p_{n} \rightarrow f(w)-w$ and $x_{n}-p_{n} \rightarrow 0$, taking the limit in (3.5), we immediately get $x_{n} \rightarrow w$. Hence $w \in \omega_{s}\left(x_{s, t_{s}}\right)$.

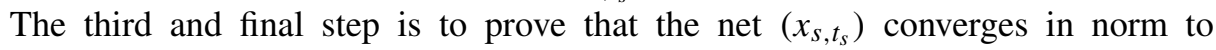
$x_{\infty}=\left(P_{S} f\right) x_{\infty}$. It suffices to prove that each norm limit point $w \in \omega_{s}\left(x_{s, t_{s}}\right)$ solves the VI (1.3). We still use the same subsequence $\left\{x_{n}\right\}$ of the net $\left(x_{s, t_{s}}\right)$ such that $x_{n} \rightarrow w$ as shown in the second step. On the other hand, for every $p \in S$, by condition (A2), we have, for each $n, p_{t_{n}} \in \operatorname{Fix}\left(W_{t_{n}}\right)$ such that $p_{t_{n}} \rightarrow p$ as $n \rightarrow \infty$.

Now since $I-W_{t_{n}}$ is monotone and since

$$
(I-f) x_{n}=-\frac{1-s_{n}}{s_{n}}\left(x_{n}-W_{t_{n}} x_{n}\right),
$$

we get

$$
\begin{aligned}
\left\langle(I-f) x_{n}, x_{n}-p_{t_{n}}\right\rangle & =-\frac{1-s_{n}}{s_{n}}\left\langle\left(x_{n}-W_{t_{n}} x_{n}\right), x_{n}-p_{t_{n}}\right\rangle \\
& =-\frac{1-s_{n}}{s_{n}}\left\langle\left(I-W_{t_{n}}\right) x_{n}-\left(I-W_{t_{n}}\right) p_{t_{n}}, x_{n}-p_{t_{n}}\right\rangle \\
& \leq 0 .
\end{aligned}
$$

Passing to the limit as $n \rightarrow \infty$ in the last inequality, we conclude that

$$
\langle(I-f) w, w-p\rangle \leq 0, \quad p \in S .
$$

This is the VI (1.3). Hence $w=x_{\infty}$, as required.

\section{References}

[1] A. Cabot, 'A proximal point algorithm controlled by a slowly vanishing term: applications to hierarchical minimization', SIAM J. Optim. 15 (2005), 555-572.

[2] K. Goebel and W. A. Kirk, Topics in Metric Fixed Point Theory, Cambridge Studies in Advanced Mathematics, 28 (Cambridge University Press, Cambridge, 1990). 
[3] Z-Q. Luo, J.-S. Pang and D. Ralph, Mathematical Programs with Equilibrium Constraints (Cambridge University Press, Cambridge, 1996).

[4] P. E. Maingé and A. Moudafi, 'Strong convergence of an iterative method for hierarchical fixed point problems', Pac. J. Optim. 3(3) (2007), 529-538.

[5] G. Marino and H. K. Xu, 'A general iterative method for nonexpansive mappings in Hilbert spaces', J. Math. Anal. Appl. 318(1) (2006), 43-52.

[6] A. Moudafi, 'Viscosity approximation methods for fixed-points problems', J. Math. Anal. Appl. 241 (2000), 46-55.

[7] A. Moudafi and P. E. Maingé, 'Towards viscosity approximations of hierarchical fixed-point problems', Fixed Point Theory Appl. (2006), Art. ID 95453, 10pp.

[8] H. K. Xu, 'An iterative approach to quadratic optimization', J. Optim. Theory Appl. 116(3) (2003), 659-678.

[9] - 'Viscosity approximation methods for nonexpansive mappings', J. Math. Anal. Appl. 298 (2004), 279-291.

[10] 'Viscosity method for hierarchical fixed point approach to variational inequalities', Taiwanese J. Math. 13(6) (2009).

[11] I. Yamada, 'The hybrid steepest descent method for the variational inequality problem over the intersection of fixed point sets of nonexpansive mappings', in: Inherently Parallel Algorithms in Feasibility and Optimization and their Applications, Studies in Computational Mathematics, 8 (eds. D. Butnariu, Y. Censor and S. Reich) (North-Holland, Amsterdam, 2001), pp. 473-504.

[12] Y. Yao and Y. C. Liou, 'Weak and strong convergence of Krasnoselski-Mann iteration for hierarchical fixed point problems', Inverse Problems 24 (2008), 501-508.

FILOMENA CIANCIARUSO, Dipartimento di Matematica, Universitá della Calabria, 87036 Arcavacata di Rende (CS), Italy e-mail: cianciaruso@unical.it

VITTORIO COLAO, Dipartimento di Matematica, Universitá della Calabria, 87036 Arcavacata di Rende (CS), Italy

e-mail: colao@mat.unical.it

LUIGI MUGLIA, Dipartimento di Matematica, Universitá della Calabria, 87036 Arcavacata di Rende (CS), Italy e-mail:muglia@mat.unical.it

HONG-KUN XU, Department of Applied Mathematics, National Sun Yat-Sen University, Kaohsiung 80424, Taiwan e-mail: xuhk@math.nsysu.edu.tw 